\title{
Almost intersecting families
}

\author{
Peter Frankl \\ Rényi Institute \\ Budapest, Hungary and \\ MIPT \\ Moscow, Russia \\ peter.frankl@gmail.com
}

\author{
Andrey Kupavskii \\ G-SCOP \\ CNRS, Université Grenobles-Alpes \\ Grenoble, France and \\ MIPT \\ Moscow, Russia \\ kupavskii@ya.ru
}

Submitted: May 25, 2020; Accepted: Mar 22, 2021; Published: Apr 9, 2021

(C) The authors. Released under the CC BY-ND license (International 4.0).

\begin{abstract}
Let $n>k>1$ be integers, $[n]=\{1, \ldots, n\}$. Let $\mathcal{F}$ be a family of $k$-subsets of $[n]$. The family $\mathcal{F}$ is called intersecting if $F \cap F^{\prime} \neq \varnothing$ for all $F, F^{\prime} \in \mathcal{F}$. It is called almost intersecting if it is not intersecting but to every $F \in \mathcal{F}$ there is at most one $F^{\prime} \in \mathcal{F}$ satisfying $F \cap F^{\prime}=\varnothing$. Gerbner et al. proved that if $n \geqslant 2 k+2$ then $|\mathcal{F}| \leqslant\left(\begin{array}{l}n-1 \\ k-1\end{array}\right)$ holds for almost intersecting families. Our main result implies the considerably stronger and best possible bound $|\mathcal{F}| \leqslant\left(\begin{array}{c}n-1 \\ k-1\end{array}\right)-\left(\begin{array}{c}n-k-1 \\ k-1\end{array}\right)+2$ for $n>(2+o(1)) k, k \geqslant 3$.
\end{abstract}

Mathematics Subject Classifications: 05D05

\section{Introduction}

Let $[n]=\{1, \ldots, n\}$ be the standard $n$-element set, $2^{[n]}$ its power set and $\left(\begin{array}{c}{[n]} \\ k\end{array}\right)$ the collection of all its $k$-subsets. Subsets of $2^{[n]}$ are called families.

A family $\mathcal{F}$ is called intersecting if $F \cap G \neq \varnothing$ for all $F, G \in \mathcal{F}$. One of the fundamental results in extremal set theory is the Erdös-Ko-Rado Theorem:

Theorem 1 ([EKR]). Suppose that $\mathcal{F} \subset\left(\begin{array}{c}{[n]} \\ k\end{array}\right)$ is intersecting, $n \geqslant 2 k>0$. Then

$$
|\mathcal{F}| \leqslant\left(\begin{array}{l}
n-1 \\
k-1
\end{array}\right)
$$

Gerbner et al. [GLPPS] proved an interesting generalisation of (1). To state it we need a definition.

Definition 2. A family $\mathcal{F} \subset 2^{[n]}$ is called almost intersecting if it is not intersecting, but to every $F \in \mathcal{F}$ there is at most one $G \in \mathcal{F}$ satisfying $F \cap G=\varnothing$. 
Theorem 3 ([GLPPS]). Suppose that $n \geqslant 2 k+2, k \geqslant 1, \mathcal{F} \subset\left(\begin{array}{c}{[n]} \\ k\end{array}\right)$. If $\mathcal{F}$ is intersecting or almost intersecting then (1) holds.

A natural example of almost intersecting families is $\left(\begin{array}{c}{[2 k]} \\ k\end{array}\right)$. For $n=2 k$ and $2 k+1$ the best possible bound $|\mathcal{F}| \leqslant\left(\begin{array}{c}2 k \\ k\end{array}\right)$ is proven in [GLPPS].

To present another example let us first define some $k$-uniform intersecting families. For integers $1 \leqslant a \leqslant b \leqslant n$ set $[a, b]=\{a, a+1, \ldots, b\}$. For a fixed $x \in[n]$ let $\mathcal{S}=\mathcal{S}(n, k, x)$ be the full star with center in $x$, i.e., $\mathcal{S}=\left\{S \in\left(\begin{array}{c}{[n]} \\ k\end{array}\right): x \in S\right\}$. Every non-empty family $\mathcal{F} \subset \mathcal{S}$ for some $x$ is called a star.

For $3 \leqslant r \leqslant k+1$ let us define

$$
\begin{aligned}
\mathcal{B}_{r}=\mathcal{B}_{r}(n, k)= & \left\{B \in\left(\begin{array}{c}
{[n]} \\
k
\end{array}\right): 1 \in B, B \cap[2, r] \neq \varnothing\right\} \cup \\
& \cup\left\{B \in\left(\begin{array}{c}
{[n]} \\
k
\end{array}\right): 1 \notin B,[2, r] \subset B\right\} .
\end{aligned}
$$

Obviously, $\left|B_{r}\right|=\left(\begin{array}{l}n-1 \\ k-1\end{array}\right)-\left(\begin{array}{c}n-r \\ k-1\end{array}\right)+\left(\begin{array}{c}n-r \\ k-r+1\end{array}\right)$. In particular, $\left|\mathcal{B}_{3}\right|=\left|\mathcal{B}_{4}\right|$. For $n>2 k$ one has

$$
\left|\mathcal{B}_{4}\right|<\left|\mathcal{B}_{5}\right|<\ldots<\left|\mathcal{B}_{k+1}\right|
$$

The family $\mathcal{B}_{k+1}$ is called the Hilton-Milner family. It has a single set, namely $[2, k+1]$, which does not contain 1 .

For $x, y \in[n]$ let us recall the standard notation:

$$
\begin{aligned}
\mathcal{F}(x) & =\{F \backslash\{x\}: x \in F \in \mathcal{F}\}, \mathcal{F}(\bar{x})=\{F \in \mathcal{F}: x \notin F\}, \\
\mathcal{F}(x, \bar{y}) & =\mathcal{F}(\bar{y}, x)=\{F \backslash\{x\}: x \in F \in \mathcal{F}, y \notin F\} .
\end{aligned}
$$

The maximum degree $\Delta(\mathcal{F})$ of a family $\mathcal{F} \subset 2^{[n]}$ is $\max \{|\mathcal{F}(x)|: x \in[n]\}$. For $3 \leqslant r \leqslant$ $k+1$,

$$
\Delta\left(\mathcal{B}_{r}\right)=\left(\begin{array}{l}
n-1 \\
k-1
\end{array}\right)-\left(\begin{array}{l}
n-r \\
k-1
\end{array}\right)=\left(\begin{array}{l}
n-2 \\
k-2
\end{array}\right)+\ldots+\left(\begin{array}{c}
n-r \\
k-2
\end{array}\right)=\left|\mathcal{B}_{r}(1)\right|
$$

Hilton and Milner [HM] proved the following stability result for intersecting families. (This theorem has many proofs, see e.g. [KZ].)

Theorem $4([\mathrm{HM}])$. Suppose that $n>2 k \geqslant 4, \mathcal{F} \subset\left(\begin{array}{c}{[n]} \\ k\end{array}\right)$ is intersecting, but $\mathcal{F}$ is not a star (not contained in a full star). Then

$$
|\mathcal{F}| \leqslant\left|\mathcal{B}_{k+1}\right|
$$

moreover, equality holds only if $\mathcal{F}$ is isomorphic to $\mathcal{B}_{k+1}$ or $k=3$ and $\mathcal{F}$ is isomorphic to $\mathcal{B}_{3}$.

Example 5. Let $B \subset\left(\begin{array}{c}{[n]} \\ k\end{array}\right)$ be an arbitrary set satisfying $1 \in B, B \cap[2, k+1]=\varnothing$. Set $\mathcal{B}^{+}=\mathcal{B}_{k+1} \cup\{B\}$. Then $\left|\mathcal{B}^{+}\right|=\left|\mathcal{B}_{k+1}\right|+1$ and $\mathcal{B}^{+}$is almost intersecting. 
Our main result is the following.

Theorem 6. Suppose that $\mathcal{F} \subset\left(\begin{array}{c}{[n]} \\ k\end{array}\right)$ is almost intersecting, $k \geqslant 3$. Then

$$
|\mathcal{F}| \leqslant\left|\mathcal{B}^{+}\right|=\left(\begin{array}{l}
n-1 \\
k-1
\end{array}\right)-\left(\begin{array}{c}
n-k-1 \\
k-1
\end{array}\right)+2
$$

holds in the following cases:

(i) $k=3, n \geqslant 13$,

(ii) $k \geqslant 4, n \geqslant 3 k+3$,

(iii) $k \geqslant 10, n>2 k+2 \sqrt{k}+4$.

Moreover, equality in (3) is only possible when $\mathcal{F}$ is isomorphic to $\mathcal{B}^{+}$.

In what follows, we omit floor and ceiling signs whenever they do not affect the calculations.

The case $k=2$ is easy. Suppose that $\mathcal{G} \subset\left(\begin{array}{c}{[n]} \\ 2\end{array}\right)$ is almost intersecting and let $F, G \in \mathcal{G}$ be pairwise disjoint. Set $X=F \cup G$ and note $|X|=4$.

Claim 7. $\mathcal{G} \subset\left(\begin{array}{c}X \\ 2\end{array}\right)$.

Proof. If $\mathcal{G}=\{F, G\}$ then we have nothing to prove. On the other hand, for any further edge $H \in \mathcal{G}$, both $F \cap H$ and $G \cap H$ must be non-empty. Since $|H|=2, H \subset X$ follows.

Note that the family $\left(\begin{array}{c}{[4]} \\ 2\end{array}\right)$ is the (unique, up to a permutation) extremal example in this case.

Let us make two simple but important observations.

Proposition 8. Let $\mathcal{F} \subset\left(\begin{array}{c}{[n]} \\ k\end{array}\right)$ be almost intersecting. Then there is a unique partition $\mathcal{F}=\mathcal{F}_{0} \sqcup \mathcal{P}_{1} \sqcup \ldots \sqcup \mathcal{P}_{\ell}$ where $\mathcal{F}_{0}$ is intersecting $\left(\mathcal{F}_{0}=\varnothing\right.$ is allowed) and for $1 \leqslant i \leqslant \ell$, $\mathcal{P}_{i}=\left\{P_{i}, Q_{i}\right\}$ with $P_{i} \cap Q_{i}=\varnothing$.

The above partition of $\mathcal{F}$ is called the canonical partition. The function $\ell(\mathcal{F})=\ell$ is an important parameter of $\mathcal{F}$.

Definition 9. A family $\mathcal{T}=\left\{T_{1}, \ldots, T_{\ell}\right\}$ satisfying $T_{i} \in \mathcal{P}_{i}$, is called a full tail (of $\mathcal{F}$ ).

Proposition 10. There are $2^{\ell}$ full tails $\mathcal{T}$ and for each of them $\mathcal{F}_{0} \cup \mathcal{T}$ is intersecting.

Let us close this section by a short proof of $(3)$ for the special case $\ell(\mathcal{F})=1$.

There are two cases to consider according whether the families $\mathcal{F}_{0} \cup\left\{P_{1}\right\}, \mathcal{F}_{0} \cup\left\{Q_{1}\right\}$ are stars or not. Suppose first that one of them, say $\mathcal{F}_{0} \cup\left\{P_{1}\right\}$ is not a star. By Theorem 4 , $\left|\mathcal{F}_{0} \cup\left\{P_{1}\right\}\right|=|\mathcal{F}|-1 \leqslant\left|\mathcal{B}_{k+1}\right|$, implying (3). For $k \geqslant 4$ uniqueness in the Hilton-Milner Theorem implies uniqueness in Theorem 6 as well. In the case $k=3$, one has the extra 
possibility $\mathcal{F}_{0} \cup\left\{P_{1}\right\}=\mathcal{B}_{3}$. However, it is easy to check that adding a new 3 -set to $\mathcal{B}_{3}$ will never produce an almost intersecting family.

The second case is even easier. If both $\mathcal{F}_{0} \cup\left\{P_{1}\right\}$ and $\mathcal{F}_{0} \cup\left\{Q_{1}\right\}$ are stars then $P_{1} \cap Q_{1}=\varnothing$ implies that there are two distinct elements (the centres of the stars) $x, y$ such that $\{x, y\} \subset F$ for all $F \in \mathcal{F}_{0}$. Consequently,

$$
|\mathcal{F}|=\left|\mathcal{F}_{0}\right|+2 \leqslant\left(\begin{array}{l}
n-2 \\
k-2
\end{array}\right)+2 \leqslant\left(\begin{array}{l}
n-2 \\
k-2
\end{array}\right)+2\left(\begin{array}{c}
n-3 \\
k-2
\end{array}\right)=\left|\mathcal{B}_{3}\right| \leqslant\left|\mathcal{B}_{k+1}\right|<\left|\mathcal{B}^{+}\right| .
$$

\section{Preliminaries}

Let us first prove an inequality on the size $\ell=\ell(\mathcal{F})$ of full tails.

\section{Proposition 11.}

$$
\ell(\mathcal{F}) \leqslant\left(\begin{array}{c}
2 k-1 \\
k-1
\end{array}\right)
$$

The proof of (4) depends on a classical result of Bollobás [B].

Theorem 12 ([B], cf. also [JP] and [Ka1]). Suppose that $a, b$ are positive integers, $\mathcal{A}=$ $\left\{A_{1}, \ldots, A_{m}\right\}, \mathcal{B}=\left\{B_{1}, \ldots, B_{m}\right\}$ are families satisfying $\left|A_{i}\right|=a,\left|B_{i}\right|=b, A_{i} \cap B_{i}=\varnothing$ for $1 \leqslant i \leqslant m$ and also

$$
A_{i} \cap B_{j} \neq \varnothing \quad \text { for all } \quad 1 \leqslant i \neq j \leqslant m \text {. }
$$

Then

$$
m \leqslant\left(\begin{array}{c}
a+b \\
a
\end{array}\right)
$$

Proof of Proposition 11. Define $A_{i}=P_{i}$ for $1 \leqslant i \leqslant \ell, A_{i}=Q_{i-\ell}$ for $\ell+1 \leqslant i \leqslant 2 \ell$ and similarly $B_{i}=Q_{i}$ for $1 \leqslant i \leqslant \ell, B_{i}=P_{i-\ell}$ for $\ell+1 \leqslant i \leqslant 2 \ell$. Then $\mathcal{A}=\left\{A_{1}, \ldots, A_{2 \ell}\right\}$ and $\mathcal{B}=\left\{B_{1}, \ldots, B_{2 \ell}\right\}$ satisfy the conditions of Theorem 12 with $a=b=k$. Thus $2 \ell \leqslant\left(\begin{array}{c}2 k \\ k\end{array}\right)$ and thereby (4) follows.

If $\mathcal{F}_{0} \neq \varnothing$, then one can use an extension (cf. [F1]) of (6) to show that (4) is strict.

Another ingredient of the proof of Theorem 6 is the following

Theorem 13 ([F2]). Suppose that $\mathcal{A} \subset\left(\begin{array}{c}{[n]} \\ k\end{array}\right), n>2 k \geqslant 6$. Let $r$ be an integer, $4 \leqslant r \leqslant$ $k+1$. If $\mathcal{A}$ is intersecting and $\Delta(\mathcal{A}) \leqslant \Delta\left(\mathcal{B}_{r}\right)$ then

$$
|\mathcal{A}| \leqslant\left|\mathcal{B}_{r}\right|
$$

See $[\mathrm{KZ}]$ for an alternative proof of this theorem.

Let us note that if $\mathcal{A}$ is not a star then for all $x \in[n]$ there exists $A(x) \in \mathcal{A}$ with $x \notin A(x)$. There are only $\left(\begin{array}{c}n-1 \\ k-1\end{array}\right)-\left(\begin{array}{c}n-k-1 \\ k-1\end{array}\right)$ sets $A \in\left(\begin{array}{c}{[n]} \\ k\end{array}\right)$ satisfying $x \in A, A \cap A(x) \neq \varnothing$. Thus $|\mathcal{A}(x)| \leqslant\left(\begin{array}{c}n-1 \\ k-1\end{array}\right)-\left(\begin{array}{c}n-k-1 \\ k-1\end{array}\right)=\left|\mathcal{B}_{k+1}(1)\right|$. This shows that Theorem 13 extends the Hilton-Milner Theorem. 
The last ingredient of the proof is the Kruskal-Katona Theorem ([Kr], [Ka2]). We use it in a form proposed by Hilton $[\mathrm{H}]$.

For fixed $n$ and $k$ let us define the lexicographic order $<_{L}$ on $\left(\begin{array}{c}{[n]} \\ k\end{array}\right)$ by setting

$$
A<_{L} B \quad \text { iff } \quad \min \{x \in A \backslash B\}<\min \{x \in B \backslash A\} .
$$

For an integer $1 \leqslant m \leqslant\left(\begin{array}{l}n \\ k\end{array}\right)$ let $\mathcal{L}(m)=\mathcal{L}(m, n, k)$ denote the family of the first $m$ subsets $A \in\left(\begin{array}{c}{[n]} \\ k\end{array}\right)$ in the lexicographic order.

Let $a, b$ be positive integers, $a+b \leqslant n$. Two families $\mathcal{A} \subset\left(\begin{array}{c}{[n]} \\ a\end{array}\right), \mathcal{B} \subset\left(\begin{array}{c}{[n]} \\ b\end{array}\right)$ are called cross-intersecting if $A \cap B \neq \varnothing$ for all $A \in \mathcal{A}, B \in \mathcal{B}$.

Theorem $14([\mathrm{Kr}],[\mathrm{Ka} 2],[\mathrm{H}])$. Let $X \subset[n]$ and $|X| \geqslant a+b$. If $\mathcal{A} \subset\left(\begin{array}{l}X \\ a\end{array}\right)$ and $\mathcal{B} \subset\left(\begin{array}{l}X \\ b\end{array}\right)$ are cross-intersecting then $\mathcal{L}(|\mathcal{A}|, X, a)$ and $\mathcal{L}(|\mathcal{B}|, X, b)$ are cross-intersecting as well.

Let us sketch the proof of this for completeness. Take the family $\mathcal{A}^{c}:=\left\{X \in\left(\begin{array}{c}{[n]} \\ n-a\end{array}\right)\right.$ : $\bar{X} \notin \mathcal{A}$ \}. Consider the $b$-shadow $\partial^{b}\left(\mathcal{A}^{c}\right)$, consisting of all sets of size $b$ that are contained in some set from $\mathcal{A}^{c}$. Then it is easy to see that $\partial^{b}\left(\mathcal{A}^{c}\right)$ must be disjoint from $\mathcal{B}$. Since the shadow of $\mathcal{A}^{c}$ is minimized for the last $\left|\mathcal{A}^{c}\right|$ sets in the lex order (which is up to a reordering of the ground set is the same as the first $\left|\mathcal{A}^{c}\right|$ sets in the colex order), the "best" choice for $\mathcal{A}$ is the family $\mathcal{L}(|\mathcal{A}|, X, a)$. And then we naturally get that $\mathcal{B}$ can be taken to be $\mathcal{L}(|\mathcal{B}|, X, b)$.

Note that if $\mathcal{G} \subset\left(\begin{array}{c}{[n]} \\ k\end{array}\right)$ is intersecting then the two families $\mathcal{G}(1) \subset\left(\begin{array}{l}{[2, n]} \\ k-1\end{array}\right)$ and $\mathcal{G}(\overline{1}) \subset$ $\left(\begin{array}{c}{[2, n]} \\ k\end{array}\right)$ are cross-intersecting. Usually we apply Theorem 14 to these families (with $X=$ $[2, n])$.

In our situation with $\mathcal{F} \subset\left(\begin{array}{c}{[n]} \\ k\end{array}\right)$ being almost intersecting and $\mathcal{F}_{0} \subset \mathcal{F}$ defined by Proposition $8, \mathcal{F}_{0}(1)$ and $\mathcal{F}(\overline{1})$ are cross-intersecting.

Using Theorem 14 one easily deduces the following.

Corollary 15. Let $r \geqslant 3$ be an integer. Suppose that $\mathcal{A} \subset\left(\begin{array}{c}{[2, n]} \\ k-1\end{array}\right)$ and $\mathcal{B} \subset\left(\begin{array}{c}{[2, n]} \\ k\end{array}\right)$ are cross-intersecting, $n>2 k, k \geqslant r$. If

$$
|\mathcal{A}| \geqslant\left(\begin{array}{l}
n-1 \\
k-1
\end{array}\right)-\left(\begin{array}{l}
n-r \\
k-1
\end{array}\right)
$$

Then

$$
|\mathcal{B}| \leqslant\left(\begin{array}{c}
n-r \\
k-r+1
\end{array}\right)
$$

Proof. Note that $\mathcal{L}\left(\left(\begin{array}{l}n-1 \\ k-1\end{array}\right)-\left(\begin{array}{c}n-r \\ k-1\end{array}\right),[2, n], k-1\right)=\left\{L \in\left(\begin{array}{c}{[2, n]} \\ k-1\end{array}\right): L \cap[2, r] \neq \varnothing\right\}$. Since $n>2 k,[2, r] \subset B$ must hold for every $B \in\left(\begin{array}{c}{[2, n]} \\ k\end{array}\right)$ which intersects each member of $\mathcal{L}\left(\left(\begin{array}{l}n-1 \\ k-1\end{array}\right)-\left(\begin{array}{l}n-r \\ k-1\end{array}\right),[2, n], k-1\right)$. Via Theorem 14 this implies $(9)$.

Corollary 16. Suppose that $\mathcal{A} \subset\left(\begin{array}{c}{[2, n]} \\ k-1\end{array}\right), \mathcal{B} \subset\left(\begin{array}{c}{[2, n]} \\ k\end{array}\right)$ are cross-intersecting, $n>2 k>2$,

$$
|\mathcal{B}| \geqslant k \text {. }
$$

Then

$$
|\mathcal{A}| \leqslant\left(\begin{array}{l}
n-1 \\
k-1
\end{array}\right)-\left(\begin{array}{l}
n-k \\
k-1
\end{array}\right)
$$


Proof. Just note that $\mathcal{L}(k,[2, n], k)=\{[2, k] \cup\{j\}, k+1 \leqslant j \leqslant 2 k\}$ and the only $(k-1)$ sets intersecting each of these $k$-sets are those which intersect $[2, k]$.

\section{Some inequalities concerning binomial coefficients}

In this section we present some inequalities that we use in Section 5. The proofs are via standard manipulations, the reader might just glance through them briefly.

\section{Lemma 17.}

$$
\begin{gathered}
\left(\begin{array}{c}
2 k \\
k-2
\end{array}\right) \geqslant\left(\begin{array}{c}
2 k-1 \\
k-1
\end{array}\right) \quad \text { for } k \geqslant 6, \\
\left(\begin{array}{c}
2 k+1 \\
k-2
\end{array}\right) \geqslant\left(\begin{array}{c}
2 k-1 \\
k-1
\end{array}\right) \quad \text { for } k \geqslant 4 .
\end{gathered}
$$

Proof. $\left(\begin{array}{c}2 k \\ k-2\end{array}\right) /\left(\begin{array}{c}2 k-1 \\ k-1\end{array}\right)=\frac{2 k \cdot(k-1)}{(k+1)(k+2)}$ which is a monotone increasing function of $k$. Since for $k=6,2 \times 6 \times 5=60>56=7 \times 8,(12)$ is proved. To prove $(13)$ just note $\left(\begin{array}{c}2 k+1 \\ k-2\end{array}\right)>\left(\begin{array}{c}2 k \\ k-2\end{array}\right)$ and check it for $k=4$ and 5 .

Lemma 18. Suppose that $k \geqslant 10$ and $3 k+2 \geqslant m \geqslant 2 k-4$. Then

$$
2 \geqslant\left(\begin{array}{c}
m \\
k-2
\end{array}\right) /\left(\begin{array}{c}
m-1 \\
k-2
\end{array}\right) \geqslant 4 / 3
$$

Moreover, if $m-s \geqslant 2 k-4$ then

$$
\sum_{0 \leqslant i \leqslant s}\left(\begin{array}{c}
m-i \\
k-2
\end{array}\right) \geqslant\left(2-\frac{1}{2^{s}}\right)\left(\begin{array}{c}
m \\
k-2
\end{array}\right) .
$$

Proof. $\left(\begin{array}{c}m \\ k-2\end{array}\right) /\left(\begin{array}{c}m-1 \\ k-2\end{array}\right)=\frac{m}{m-k+2}$. Now (14) is equivalent to

$$
2 m-2 k+4 \geqslant m \geqslant \frac{4}{3} m-\frac{4}{3} k+\frac{8}{3} .
$$

The first part is equivalent to $m \geqslant 2 k-4$, the second to $4 k-8 \geqslant m$. As for $k \geqslant 10$, $4 k-8 \geqslant 3 k+2$, we are done. The inequality (15) is a direct application of (14).

Lemma 19. Suppose that $n \geqslant 2(k+\sqrt{k}+2), k \geqslant 9, r \geqslant \sqrt{k}+5$. Then

$$
\left(\begin{array}{c}
n-r+1 \\
k-r+2
\end{array}\right)<\left(\begin{array}{c}
n-r-1 \\
k-2
\end{array}\right) \text {. }
$$

Proof. Let us first show that for $n, k$ fixed the function $f(r)=\left(\begin{array}{c}n-r+1 \\ k-r+2\end{array}\right) /\left(\begin{array}{c}n-r-1 \\ k-2\end{array}\right)$ is monotone decreasing in $r$. Indeed, $f(r+1) / f(r)=\frac{n-r-1}{n-r+1} \cdot \frac{k-r+2}{n-k-r+1}<1$ as both factors are less than 1 for $n>2 k+1$.

Consequently it is sufficient to check (16) in the case $r=t+1$ where $t=\lfloor\sqrt{k}\rfloor+4$. Fixing $k$ and thereby $r, t$, define

$$
g(n)=\left(\begin{array}{c}
n-t \\
k-t+1
\end{array}\right) /\left(\begin{array}{c}
n-t-2 \\
k-2
\end{array}\right) .
$$


Claim 20. For $n \in \mathbb{R}$ and $n \geqslant 2 k, g(n)$ is a monotone decreasing function of $n$.

Proof. Indeed,

$$
g(n+1) / g(n)=\frac{n-t+1}{n-t-1} \cdot \frac{n-k-t+1}{n-k} \leqslant \frac{(n-t+1)(n-k-2)}{(n-t-1)(n-k)}<1
$$

where we used $t \geqslant 3$ and $a b>(a-2)(b+2)$ for $a>b+2>0$.

In view of the claim it is sufficient to prove (16) for the case $n=2 k+2 \sqrt{k}+4$.

$$
\frac{\left(\begin{array}{c}
n-t \\
k-t+1
\end{array}\right)}{\left(\begin{array}{c}
n-t-2 \\
k-2
\end{array}\right)}=\frac{(n-t)(n-t-1)}{(n-k-t+2)(n-k-t+1)} \cdot \prod_{0 \leqslant j \leqslant t-4} \frac{k-2-j}{n-k-1-j} .
$$

To estimate the RHS, note that the first part is at most $2 \times 2=4$. As to the product part, we can use the inequality $\frac{(a-i)(a+i)}{(b-i)(b+i)}<\left(\frac{a}{b}\right)^{2}$, valid for all $b>a>i>0$ to get the upper bound

$$
\left(\frac{k-\frac{t}{2}}{n-k+1-\frac{t}{2}}\right)^{t-3}=\left(1-\frac{n+1-2 k}{n-k+1-\frac{t}{2}}\right)^{t-3} .
$$

To prove (16) we need to show that this quantity is at most $1 / 4$. We show the stronger upper bound $e^{-\frac{3}{2}}$. Using the inequality $1-x<e^{-x}$, it is sufficient to show

$$
\frac{n+1-2 k}{n+1-k-\frac{t}{2}}>\frac{3}{2(t-3)}
$$

Plugging in $n=2 k+2 \sqrt{k}+4, t=\sqrt{k}+4$ the above inequality is equivalent to

$$
2(\sqrt{k}+1)(2 \sqrt{k}+5)>3 k+\frac{9}{2} \sqrt{k}+9, \quad \text { or }
$$

$k+9.5 \sqrt{k}+1>0$ which is true for $k \geqslant 0$.

Lemma 21. Suppose that $n \geqslant 3 k+3, k \geqslant 4$ then

$$
\left(\begin{array}{l}
n-4 \\
k-3
\end{array}\right)+\left(\begin{array}{c}
2 k-1 \\
k-1
\end{array}\right) \leqslant\left(\begin{array}{l}
n-5 \\
k-2
\end{array}\right)+\left(\begin{array}{l}
n-5 \\
k-4
\end{array}\right)
$$

Proof. Let us first prove (18) in the case $n=3 k+3$,

$$
\left(\begin{array}{c}
3 k-1 \\
k-3
\end{array}\right)+\left(\begin{array}{c}
2 k-1 \\
k-1
\end{array}\right) \leqslant\left(\begin{array}{c}
3 k-2 \\
k-2
\end{array}\right)+\left(\begin{array}{c}
3 k-2 \\
k-4
\end{array}\right)
$$

The cases $k=4,5,6$ can be checked directly. Let $k \geqslant 7$. Note that

$$
\left(\begin{array}{c}
3 k-1 \\
k-3
\end{array}\right) /\left(\begin{array}{c}
3 k-2 \\
k-2
\end{array}\right)=\frac{(3 k-1)(k-2)}{(2 k+1)(2 k+2)}=\frac{3 k^{2}-7 k+2}{4 k^{2}+6 k+2}<\frac{3}{4} .
$$


Thus it is sufficient to show

$$
\left(\begin{array}{c}
2 k-1 \\
k-1
\end{array}\right) /\left(\begin{array}{c}
3 k-2 \\
k-2
\end{array}\right) \leqslant \frac{1}{4}
$$

In view of $k \geqslant 7,\left(\begin{array}{c}2 k-1 \\ k-1\end{array}\right) /\left(\begin{array}{c}2 k \\ k-2\end{array}\right)$ is less than 1 . Thus (20) will follow from

$$
\left(\begin{array}{c}
2 k \\
k-2
\end{array}\right) /\left(\begin{array}{c}
2 k+4 \\
k-2
\end{array}\right)=\frac{(k+6)(k+5)(k+4)(k+3)}{(2 k+4)(2 k+3)(2 k+2)(2 k+1)}<\frac{1}{4} .
$$

Since $\frac{k+i+2}{2 k+i}=\frac{1}{2}+\frac{\frac{i}{2}+2}{2 k+i}$ is a decreasing function of $k$, it is sufficient to check (21) for $k=7$. Plugging in $k=7$ we obtain $\frac{143}{612}<\frac{1}{4}$, as desired.

To prove (18) for $n>3 k+3$, we show that passing from $n$ to $n+1$ the RHS increases more than the LHS. More exactly we show:

$$
\left(\begin{array}{l}
n-4 \\
k-4
\end{array}\right)<\left(\begin{array}{l}
n-5 \\
k-3
\end{array}\right)
$$

We have

$$
\left(\begin{array}{l}
n-4 \\
k-4
\end{array}\right) /\left(\begin{array}{l}
n-5 \\
k-3
\end{array}\right)=\frac{(n-4)(k-3)}{(n-k)(n-k-1)}
$$

Using $n>3 k, \frac{n-4}{n-k}<2$ and $\frac{k-3}{n-k-1}<\frac{1}{2}$, we get $(22)$.

\section{The case $k=3, n \geqslant 13$}

Let $\mathcal{F}=\mathcal{F}_{0} \cup \mathcal{P}_{1} \cup \ldots \cup \mathcal{P}_{\ell}$ be the canonical partition of the almost intersecting family $\mathcal{F} \subset\left(\begin{array}{c}{[n]} \\ 3\end{array}\right)$. Let us make the indirect assumption that

$$
|\mathcal{F}| \geqslant\left|\mathcal{B}^{+}\right|=\left(\begin{array}{c}
n-1 \\
2
\end{array}\right)-\left(\begin{array}{c}
n-4 \\
2
\end{array}\right)+2=3 n-7
$$

and that $\mathcal{F}$ is not isomorphic to $\mathcal{B}^{+}$. In view of $(4)$ and $2\left(\begin{array}{l}5 \\ 2\end{array}\right)=20<3 n-6$ one has $\mathcal{F}_{0} \neq \varnothing$. The proof at the end of Section 1 implies $\ell(\mathcal{F}) \geqslant 2$.

For notational convenience we set $(a, b, c)=\{a, b, c\}$. By symmetry we assume $\mathcal{P}_{1}=$ $\{(1,2,3),(4,5,6)\}$. Note that for $F \in\left(\mathcal{F} \backslash \mathcal{P}_{1}\right), F \cap(1,2,3) \neq \varnothing$ and $F \cap(4,5,6) \neq \varnothing$ imply

$$
|F \backslash[6]| \leqslant 1
$$

and

$$
\{a, b\} \subset F \quad \text { for at least one of the } 9 \text { choices } 1 \leqslant a \leqslant 3,4 \leqslant b \leqslant 6 \text {. }
$$

For $\{a, b\}, 1 \leqslant a \leqslant 3,4 \leqslant b \leqslant 6$ define $D(a, b)=\{c \in[7, n],(a, b, c) \in \mathcal{F}\}$. Let $\left(a_{1}, a_{2}, a_{3}\right)$ and $\left(b_{1}, b_{2}, b_{3}\right)$ be some permutations of $(1,2,3)$ and $(4,5,6)$, respectively. 


\section{Lemma 22.}

(i) If $D\left(a_{i}, b_{i}\right) \neq \varnothing$ for $i=1,2,3$ then $D\left(a_{i}, b_{i}\right)$ is the same 1 -element set for $1 \leqslant i \leqslant 3$.

(ii) If $\left|D\left(a_{1}, b_{1}\right)\right| \geqslant 3$ then $D\left(a_{i}, b_{i}\right)=\varnothing$ for $i=2,3$.

Proof. Suppose by symmetry $\left|D\left(a_{1}, b_{1}\right)\right| \geqslant 2$ and let $x, y \in D\left(a_{1}, b_{1}\right)$. The almost intersecting property implies $\left(a_{i}, b_{i}, z\right) \notin \mathcal{F}$ for $i=2,3$ and $z \notin\{x, y\}$. This already proves (ii). To continue with the proof of (i) choose $x_{2}, x_{3} \in\{x, y\}$, not necessarily distinct elements so that $\left(a_{i}, b_{i}, x_{i}\right) \in \mathcal{F}$ for $i=2,3$.

There are two simple cases to consider. Either $x_{2}=x_{3}$ or $x_{2} \neq x_{3}$. By symmetry assume $x_{3}=y$. In the first case $\left(a_{1}, b_{1}, x\right)$ is disjoint to both $\left(a_{2}, b_{2}, y\right)$ and $\left(a_{3}, b_{3}, y\right)$. While in the latter case $\left(a_{3}, b_{3}, y\right)$ is disjoint to both $\left(a_{1}, b_{1}, x\right)$ and $\left(a_{2}, b_{2}, x\right)$. These contradict the almost intersecting property.

Lemma 23. If $|D(a, b)| \geqslant 3$ for some $1 \leqslant a \leqslant 3,4 \leqslant b \leqslant 6$, then $\{a, b\} \cap F \neq \varnothing$ for all $F \in \mathcal{F}$.

Proof. Suppose by symmetry $(a, b)=(1,4)$ and $(1,4, c) \in \mathcal{F}$ for $c=7,8,9$. Let indirectly $F \in \mathcal{F}$ satisfy $F \cap\{1,4\}=\varnothing$. By $(24),|F \cap(7,8,9)| \leqslant 1$. Thus $F$ is disjoint to at least two of the three triples $(1,4, c), 7 \leqslant c \leqslant 9$, the desired contradiction.

How many choices of $(a, b), 1 \leqslant a \leqslant 3,4 \leqslant b \leqslant 6$ can be that satisfy $|D(a, b)| \geqslant 3$ ? In view of Lemma 22 (ii), $\{a, b\} \cap\left\{a^{\prime}, b^{\prime}\right\} \neq \varnothing$ must hold for distinct choices. Recall the easy fact that every bipartite graph without two disjoint edges is a star. Apply this on the bipartite graph with two classes $\{1,2,3\}$ and $\{4,5,6\}$ and edges corresponding to pairs $(a, b)$ with $|D(a, b)| \geqslant 3$ and get that all of these edges share a common vertex. Consequently, by symmetry, we may assume that $|D(a, b)| \geqslant 3$ implies $a=1$. Let us distinguish four cases.

$$
|D(1, j)| \geqslant 3 \quad \text { for } \quad j=4,5,6 .
$$

We claim that $\mathcal{F}(\overline{1})=\{(4,5,6)\}$. Let us prove it. Suppose that $F \in \mathcal{F}, 1 \notin F$ and by symmetry $4 \notin F$. Choose $(x, y, z) \subset[7, n]$ such that $(1,4, x),(1,4, y),(1,4, z) \in \mathcal{F}$. In view of (24) at least two of them are disjoint to $F$, a contradiction.

Since $(1,2,3)$ is the only member of $\mathcal{F}$ disjoint to $(4,5,6)$, now $\mathcal{F} \subset\{(1, u, v)$ : $\{u, v\} \cap(4,5,6) \neq \varnothing\} \cup\{(1,2,3),(4,5,6)\}$ follows.

$$
|D(1, j)| \geqslant 3 \quad \text { for } \quad j=4,5, \quad \text { but } \quad|D(1,6)| \leqslant 2 .
$$

In view of Lemma 22 (ii), $D(a, b)=\varnothing$ for $a=2,3$ and $b=4,5,6$. Using (24) as well we infer

$$
\left|\mathcal{F} \backslash\left(\begin{array}{c}
{[6]} \\
3
\end{array}\right)\right| \leqslant 2(n-6)+|D(1,6)| .
$$

To estimate $\left|\mathcal{F} \cap\left(\begin{array}{c}{[6]} \\ 3\end{array}\right)\right|$ we need another simple lemma. 
Lemma 24. If $|D(a, b)| \geqslant 2$ for some $1 \leqslant a \leqslant 3,4 \leqslant b \leqslant 6$ then $[6] \backslash\{a, b\}$ contains no member of $\mathcal{F}$.

Proof. If $E \in\left(\begin{array}{c}{[6] \backslash\{a, b\}} \\ 3\end{array}\right)$, then $E \cap(a, b, c)=\varnothing$ for all $c \in D(a, b)$. Thus almost intersection implies $E \notin \mathcal{F}$.

Applying the lemma to both $(a, b)=(1,4)$ and $(1,5)$ yields $\left|\mathcal{F} \cap\left(\begin{array}{c}{[6]} \\ 3\end{array}\right)\right| \leqslant 20-7=13$. In case $|D(1,6)|=2$, we have

$$
|\mathcal{F}| \leqslant 2(n-6)+2+13=2 n+3<3 n-7 \quad \text { for } \quad n \geqslant 13 .
$$

(c) $\quad|D(1,4)| \geqslant 3>|D(a, b)| \quad$ for $\quad(a, b) \neq(1,4), \quad 1 \leqslant a \leqslant 3, \quad 4 \leqslant b \leqslant 6$.

In view of Lemma 22 (ii), $D(a, b)=\varnothing$ is guaranteed if $(a, b) \cap(1,4)=\varnothing$. This leads to

$$
\left|\mathcal{F} \backslash\left(\begin{array}{c}
{[6]} \\
3
\end{array}\right)\right| \leqslant n-6+4 \times 2=n+2 .
$$

On the other hand Lemma 24 yields

$$
\left|\mathcal{F} \cap\left(\begin{array}{c}
{[6]} \\
3
\end{array}\right)\right| \leqslant 20-4=16 .
$$

Together with (27) this implies

$$
|\mathcal{F}| \leqslant n+18<3 n-7 \quad \text { for } \quad n \geqslant 13
$$

(d)

$$
|D(a, b)| \leqslant 2 \quad \text { for all } \quad(a, b), \quad 1 \leqslant a \leqslant 3, \quad 4 \leqslant b \leqslant 6 .
$$

Applying Lemma 22 (i) and (ii) gives that

$$
\left|D\left(a_{1}, b_{1}\right)\right|+\left|D\left(a_{2}, b_{2}\right)\right|+\left|D\left(a_{3}, b_{3}\right)\right| \leqslant 4
$$

Using this for three disjoint matchings from the complete bipartite graph between 1,2,3 and 4,5,6 yields

$$
\left|\mathcal{F} \backslash\left(\begin{array}{c}
{[6]} \\
3
\end{array}\right)\right| \leqslant 12
$$

Thus

$$
|\mathcal{F}| \leqslant 32 \leqslant 3 n-7 \quad \text { for } \quad n \geqslant 13 .
$$

In case of equality, $\left(\begin{array}{c}{[6]} \\ 3\end{array}\right) \subset \mathcal{F}$. However, that would immediately imply $\mathcal{F}=\left(\begin{array}{c}{[6]} \\ 3\end{array}\right)$. Thus the proof of the case $k=3, n \geqslant 13$ is complete. 


\section{The proof of (3) for $k \geqslant 4$}

We are going to distinguish three cases according to $\Delta\left(\mathcal{F}_{0}\right)$.

$$
\Delta\left(\mathcal{F}_{0}\right) \leqslant\left(\begin{array}{l}
n-2 \\
k-2
\end{array}\right)+\left(\begin{array}{l}
n-3 \\
k-2
\end{array}\right)=\left(\begin{array}{l}
n-1 \\
k-1
\end{array}\right)-\left(\begin{array}{l}
n-3 \\
k-1
\end{array}\right)
$$

Let us suppose $n \geqslant 2 k+5$. In view of (13),

$$
\left(\begin{array}{l}
n-4 \\
k-2
\end{array}\right)>\left(\begin{array}{c}
2 k-1 \\
k-1
\end{array}\right)
$$

Consequently, for any choice of a full tail $\mathcal{T}$,

$$
\Delta\left(\mathcal{F}_{0} \cup \mathcal{T}\right) \leqslant \Delta\left(\mathcal{F}_{0}\right)+\ell \leqslant\left(\begin{array}{l}
n-2 \\
k-2
\end{array}\right)+\left(\begin{array}{l}
n-3 \\
k-2
\end{array}\right)+\left(\begin{array}{l}
n-4 \\
k-2
\end{array}\right)=\left(\begin{array}{l}
n-1 \\
k-1
\end{array}\right)-\left(\begin{array}{l}
n-4 \\
k-1
\end{array}\right) .
$$

Thus we may apply (7) with $r=4$ :

$$
\left|\mathcal{F}_{0} \cup \mathcal{T}\right| \leqslant\left(\begin{array}{l}
n-1 \\
k-1
\end{array}\right)-\left(\begin{array}{l}
n-4 \\
k-1
\end{array}\right)+\left(\begin{array}{l}
n-4 \\
k-3
\end{array}\right) .
$$

From $(28)$ and $\ell \leqslant\left(\begin{array}{c}2 k-1 \\ k-1\end{array}\right)$ we infer

$$
|\mathcal{F}| \leqslant\left(\begin{array}{l}
n-1 \\
k-1
\end{array}\right)-\left(\begin{array}{l}
n-4 \\
k-1
\end{array}\right)+\left(\begin{array}{c}
n-4 \\
k-3
\end{array}\right)+\left(\begin{array}{c}
2 k-1 \\
k-1
\end{array}\right)
$$

Using $\left|\mathcal{B}^{+}\right|>\left|\mathcal{B}_{k+1}\right| \geqslant\left|\mathcal{B}_{5}\right|$, it is sufficient to show that the RHS is not larger than $\left|B_{5}\right|$. Equivalently

$$
\left(\begin{array}{l}
n-4 \\
k-3
\end{array}\right)+\left(\begin{array}{c}
2 k-1 \\
k-1
\end{array}\right) \leqslant\left(\begin{array}{l}
n-5 \\
k-2
\end{array}\right)+\left(\begin{array}{l}
n-5 \\
k-4
\end{array}\right) .
$$

Since (30) is the same as (18), for $n \geqslant 3 k+3$ we are done.

To deal with the case (iii), we cannot be so generous. We assume that $n \leqslant 3 k+2$. Note that

$$
\left|\mathcal{B}^{+}\right|>\left(\begin{array}{l}
n-1 \\
k-1
\end{array}\right)-\left(\begin{array}{c}
n-k-1 \\
k-1
\end{array}\right) \geqslant\left(\begin{array}{l}
n-1 \\
k-1
\end{array}\right)-\left(\begin{array}{c}
2 k+1 \\
k-1
\end{array}\right) .
$$

Using (29) and the inequality above, it is sufficient for us to show that

$$
\left(\begin{array}{l}
n-4 \\
k-1
\end{array}\right)-\left(\begin{array}{l}
n-4 \\
k-3
\end{array}\right) \geqslant 2\left(\begin{array}{c}
2 k+1 \\
k-1
\end{array}\right)
$$

The left hand side is

$$
\left(1-\frac{(k-1)(k-2)}{(n-k-1)(n-k-2)}\right)\left(\begin{array}{l}
n-4 \\
k-1
\end{array}\right) \geqslant\left(1-\frac{k^{2}}{(n-k)^{2}}\right)\left(\begin{array}{l}
n-4 \\
k-1
\end{array}\right) \geqslant
$$




$$
\left(1-\left(\frac{k}{k+2 \sqrt{k}+4}\right)^{2}\right)\left(\begin{array}{l}
n-4 \\
k-1
\end{array}\right) \geqslant\left(1-\left(1-\frac{2}{\sqrt{k}}+\frac{1}{k}\right)^{2}\right)\left(\begin{array}{l}
n-4 \\
k-1
\end{array}\right) \geqslant 2 k^{-1 / 2}\left(\begin{array}{l}
n-4 \\
k-1
\end{array}\right) .
$$

Thus, it is sufficient for us to show that

$$
\left(\begin{array}{l}
n-4 \\
k-1
\end{array}\right) /\left(\begin{array}{c}
2 k+1 \\
k-1
\end{array}\right) \geqslant k^{1 / 2}
$$

Let us define $2 p=n-2 k-4$ and note $p>\sqrt{k}$. In view of (14) and $n \leqslant 3 k+2$ we have

$$
\left(\begin{array}{c}
n-4 \\
k-1
\end{array}\right) /\left(\begin{array}{c}
2 k+1 \\
k-1
\end{array}\right)>(4 / 3)^{2 p-1}>p>\sqrt{k},
$$

since $(4 / 3)^{2 x-1}>x$ holds for all $x>0$. This concludes the proof of $(3)$ in this case.

$$
\left(\begin{array}{l}
n-1 \\
k-1
\end{array}\right)-\left(\begin{array}{l}
n-3 \\
k-1
\end{array}\right)<\Delta\left(\mathcal{F}_{0}\right) \leqslant\left(\begin{array}{l}
n-1 \\
k-1
\end{array}\right)-\left(\begin{array}{l}
n-k \\
k-1
\end{array}\right)
$$

Let 1 be the vertex of highest degree in $\mathcal{F}_{0}$.

Claim 25. Let $\mathcal{G} \subset\left(\begin{array}{c}{[n]} \\ k\end{array}\right)$ be any intersecting family containing $\mathcal{F}_{0}$. Then 1 is the unique vertex of highest degree in $\mathcal{G}$.

Proof. By assumption $|\mathcal{G}(1)| \geqslant\left|\mathcal{F}_{0}(1)\right|>\left(\begin{array}{l}n-2 \\ k-2\end{array}\right)+\left(\begin{array}{l}n-3 \\ k-2\end{array}\right)$.

Let $2 \leqslant x \leqslant n$ be an arbitrary vertex. In view of Corollary 15 ,

$$
|\mathcal{G}(\overline{1}, x)| \leqslant|\mathcal{G}(\overline{1})| \leqslant\left(\begin{array}{l}
n-3 \\
k-2
\end{array}\right) .
$$

The inequality

$$
|\mathcal{G}(1, x)| \leqslant\left(\begin{array}{l}
n-2 \\
k-2
\end{array}\right)
$$

is obvious. Therefore $|\mathcal{G}(x)|=|\mathcal{G}(\overline{1}, x)|+|\mathcal{G}(1, x)| \leqslant\left(\begin{array}{l}n-2 \\ k-2\end{array}\right)+\left(\begin{array}{l}n-3 \\ k-2\end{array}\right)<|\mathcal{G}(1)|$.

Define the parameter $r, 4 \leqslant r \leqslant k$ by

$$
\left(\begin{array}{l}
n-1 \\
k-1
\end{array}\right)-\left(\begin{array}{c}
n-(r-1) \\
k-1
\end{array}\right)<\Delta\left(\mathcal{F}_{0}\right) \leqslant\left(\begin{array}{l}
n-1 \\
k-1
\end{array}\right)-\left(\begin{array}{l}
n-r \\
k-1
\end{array}\right) .
$$

Let us choose the full tail $\mathcal{T}$ so that $1 \notin T$ for all $T \in \mathcal{T}$. Applying Claim 25 to $\mathcal{G}=\mathcal{F}_{0} \cup \mathcal{T}$ yields $\Delta\left(\mathcal{F}_{0} \cup \mathcal{T}\right)=\Delta\left(\mathcal{F}_{0}\right)$. Thus Theorem 13 implies

$$
\left|\mathcal{F}_{0} \cup \mathcal{T}\right| \leqslant\left(\begin{array}{l}
n-1 \\
k-1
\end{array}\right)-\left(\begin{array}{c}
n-r \\
k-1
\end{array}\right)+\left(\begin{array}{c}
n-r \\
k-r+1
\end{array}\right) .
$$

Let us first prove (3) in the case $n \geqslant 3 k+3$. Using $\left|\mathcal{B}_{r}\right| \leqslant\left|\mathcal{B}_{k}\right|$ and $\ell(\mathcal{F}) \leqslant\left(\begin{array}{c}2 k-1 \\ k-1\end{array}\right)$ it is sufficient to show $\left(\begin{array}{c}n-1 \\ k-1\end{array}\right)-\left(\begin{array}{c}n-k \\ k-1\end{array}\right)+\left(\begin{array}{c}n-k \\ 1\end{array}\right)+\left(\begin{array}{c}2 k-1 \\ k-1\end{array}\right)<\left(\begin{array}{c}n-1 \\ k-1\end{array}\right)-\left(\begin{array}{c}n-k-1 \\ k-1\end{array}\right)+2$, or equivalently 
$\left(\begin{array}{c}2 k-1 \\ k-1\end{array}\right)<\left(\begin{array}{c}n-k-1 \\ k-2\end{array}\right)-(n-k)+2$. For $n \geqslant 3 k+3$ the RHS is an increasing function of $n$. Thus it is sufficient to check the case $n=3 k+3$ :

$$
\left(\begin{array}{c}
2 k-1 \\
k-1
\end{array}\right)<\left(\begin{array}{c}
2 k+2 \\
k-2
\end{array}\right)-2 k-1=\left(\begin{array}{c}
2 k+1 \\
k-2
\end{array}\right)+\left(\left(\begin{array}{c}
2 k+1 \\
k-3
\end{array}\right)-2 k-1\right) .
$$

This inequality is true by (13) and $k-3 \geqslant 1$.

Now let us turn to the case $k \geqslant 10,3 k+2 \geqslant n \geqslant 2(k+\sqrt{k}+2)$. Recall the definition of $r$ from (32).

Using (4) and Corollary 15 we have

$$
\ell=\ell(\mathcal{F}) \leqslant \min \left\{\left(\begin{array}{c}
2 k-1 \\
k-1
\end{array}\right),\left(\begin{array}{c}
n-r+1 \\
k-r+2
\end{array}\right)\right\} .
$$

Let us first consider the case

$$
r<\sqrt{k}+5
$$

We are going to prove (3) in the form

$$
|\mathcal{F}| \leqslant\left(\begin{array}{c}
n-1 \\
k-1
\end{array}\right)-\left(\begin{array}{c}
n-r \\
k-1
\end{array}\right)+\left(\begin{array}{c}
n-r \\
k-r+1
\end{array}\right)+\left(\begin{array}{c}
2 k-1 \\
k-1
\end{array}\right) \leqslant\left(\begin{array}{c}
n-1 \\
k-1
\end{array}\right)-\left(\begin{array}{c}
n-k-1 \\
k-1
\end{array}\right)
$$

or equivalently

$$
\left(\begin{array}{c}
n-r \\
k-r+1
\end{array}\right)+\left(\begin{array}{c}
2 k-1 \\
k-1
\end{array}\right) \leqslant\left(\begin{array}{c}
n-r-1 \\
k-2
\end{array}\right)+\left(\begin{array}{c}
n-r-2 \\
k-2
\end{array}\right)+\cdots+\left(\begin{array}{c}
n-k-1 \\
k-2
\end{array}\right) .
$$

We want to apply (15) to the RHS. Note that $n-s \geqslant 2 k-4$ is satisfied if $s \leqslant 2 \sqrt{k}+8$. Since $r<\sqrt{k}+5,\left(2-2^{-\sqrt{k}}\right)\left(\begin{array}{c}n-r-1 \\ k-2\end{array}\right)$ is a lower bound for the RHS. As to $\left(\begin{array}{c}2 k-1 \\ k-1\end{array}\right)$, in view of (12) and (14) it is very small, e.g.,

$$
\left(\begin{array}{c}
2 k-1 \\
k-1
\end{array}\right)<\operatorname{RHS} \times\left(\frac{4}{3}\right)^{-\sqrt{k}}
$$

As to the main term, $\left(\begin{array}{c}n-r \\ k-r+1\end{array}\right)$, using $r \geqslant 4$ we have

$$
\begin{aligned}
\left(\begin{array}{c}
n-r \\
k-r+1
\end{array}\right) & \leqslant\left(\begin{array}{c}
n-r \\
k-3
\end{array}\right)=\left(\begin{array}{c}
n-r-1 \\
k-2
\end{array}\right) \frac{(n-r)(k-2)}{(n-r-k+3)(n-r-k+2)} \leqslant \\
& \leqslant \frac{n-4}{n-4-(k-3)} \cdot \frac{k-2}{n-4-(k-2)}\left(\begin{array}{c}
n-r-1 \\
k-2
\end{array}\right) .
\end{aligned}
$$

Both factors in the coefficient of $\left(\begin{array}{c}n-r-1 \\ k-2\end{array}\right)$ are decreasing functions of $n$. Thus the maximum is attained for $n=2 k+2 \sqrt{k}+4$ and its value is

$$
\frac{2(k+\sqrt{k})}{(k+\sqrt{k})+(\sqrt{k}+3)} \cdot \frac{k-2}{k-2+2 \sqrt{k}+2} \stackrel{\text { def }}{=} h(k) .
$$


To prove (34) it is sufficient to show

$$
h(k)+\left(\frac{4}{3}\right)^{-\sqrt{k}}<2-2^{-\sqrt{k}} .
$$

Since

$$
h(k)<\frac{2}{1+\frac{1}{\sqrt{k}}} \cdot \frac{1}{1+\frac{2}{\sqrt{k}}}<2-\frac{2}{\sqrt{k}},
$$

we are done.

Let us now suppose that $\sqrt{k}+5 \leqslant r<k$. We want to establish (3) in the form

$$
|\mathcal{F}|=\left|\mathcal{F}_{0} \cup \mathcal{T}\right|+\ell(\mathcal{F})<\left|\mathcal{B}_{r+2}\right| \text {. }
$$

Using (33) and (34) one sees that the following inequality is sufficient:

$$
\left(\begin{array}{c}
n-r \\
k-r+1
\end{array}\right)+\left(\begin{array}{c}
n-r+1 \\
k-r+2
\end{array}\right) \leqslant\left(\begin{array}{c}
n-r-1 \\
k-2
\end{array}\right)+\left(\begin{array}{c}
n-r-2 \\
k-2
\end{array}\right) .
$$

This inequality is the sum of (16) applied once for $r$ and once for $r+1$.

The final subcase is $r=k$. Using (33) and (34) we obtain

$$
|\mathcal{F}| \leqslant\left(\begin{array}{l}
n-1 \\
k-1
\end{array}\right)-\left(\begin{array}{c}
n-k \\
k-1
\end{array}\right)+\left(\begin{array}{c}
n-k \\
1
\end{array}\right)+\left(\begin{array}{c}
n-k+1 \\
2
\end{array}\right) .
$$

To show $|\mathcal{F}|<\left|\mathcal{B}^{+}\right|$it is sufficient to show

$$
\left(\begin{array}{c}
n-k \\
1
\end{array}\right)+\left(\begin{array}{c}
n-k+1 \\
2
\end{array}\right) \leqslant\left(\begin{array}{c}
n-k-1 \\
3
\end{array}\right)<\left(\begin{array}{c}
n-k-1 \\
k-2
\end{array}\right)+2 .
$$

The second half of (36) is evident from $k \geqslant 10$ and $n>2 k+4$. To show the first half note that

$$
\left(\begin{array}{c}
n-k+1 \\
1
\end{array}\right)+\left(\begin{array}{c}
n-k+1 \\
2
\end{array}\right)=\left(\begin{array}{c}
n-k+2 \\
2
\end{array}\right)<2\left(\begin{array}{c}
n-k-1 \\
2
\end{array}\right)
$$

where the last inequality is true for $n-k-1 \geqslant 8$.

On the other hand, for $n-k-1 \geqslant 8$ one has also $2\left(\begin{array}{c}n-k-1 \\ 2\end{array}\right) \leqslant\left(\begin{array}{c}n-k-1 \\ 3\end{array}\right)$, concluding the proof of $(36)$.

$$
\left(\begin{array}{l}
n-1 \\
k-1
\end{array}\right)-\left(\begin{array}{l}
n-k \\
k-1
\end{array}\right)<\Delta\left(\mathcal{F}_{0}\right)
$$

In view of Corollary 16 we have

$$
\left|\mathcal{F}_{0}(\overline{1})\right|+\ell(\mathcal{F}) \leqslant k-1 .
$$


On the other hand, having solved the case $\ell(\mathcal{F})=1$ in Section 1 , we know that $\ell(\mathcal{F}) \geqslant 2$.

The first two $k$-subsets of $\left(\begin{array}{c}{[2, n]} \\ k\end{array}\right)$ in the lexicographic order are $[2, k+1]$ and $[2, k] \cup$ $\{k+2\}$. Using Theorem 14 we infer

$$
\left|\mathcal{F}_{0}(1)\right| \leqslant\left(\begin{array}{l}
n-1 \\
k-1
\end{array}\right)-\left(\begin{array}{c}
n-k \\
k-1
\end{array}\right)+\left(\begin{array}{c}
n-k-2 \\
k-2
\end{array}\right)
$$

Adding (37), (38) and using $\ell(\mathcal{F}) \leqslant k-1$ we obtain

$$
|\mathcal{F}| \leqslant\left(\begin{array}{l}
n-1 \\
k-1
\end{array}\right)-\left(\begin{array}{c}
n-k \\
k-1
\end{array}\right)+\left(\begin{array}{c}
n-k-2 \\
k-2
\end{array}\right)+2(k-1)
$$

To prove (3) we need

$$
\left(\begin{array}{c}
n-k-2 \\
k-2
\end{array}\right)+2(k-1)<\left(\begin{array}{c}
n-k \\
k-1
\end{array}\right)-\left(\begin{array}{c}
n-k-1 \\
k-1
\end{array}\right)+2 .
$$

Rearranging yields

$$
2(k-1)<\left(\begin{array}{c}
n-k-2 \\
k-3
\end{array}\right)+2
$$

For $k=4$ this is simply

$$
6<(n-6)+2, \quad \text { i.e., } \quad n \geqslant 11 .
$$

For $k \geqslant 5, k-3 \geqslant 2$ and therefore

$$
\left(\begin{array}{c}
n-k-2 \\
2
\end{array}\right)>2(k-2) \quad \text { is sufficient. }
$$

This inequality is satisfied for $n \geqslant 2 k+2$. Indeed,

$$
\left(\begin{array}{l}
k \\
2
\end{array}\right)=\frac{k}{2}(k-1)>2(k-2) \quad \text { already for } k \geqslant 3 \text {. }
$$

This concludes the entire proof.

\section{Acknowledgements}

We thank the anonymous referees for carefully reading the paper and providing us with their comments. The authors acknowledge the financial support from the Ministry of Education and Science of the Russian Federation in the framework of MegaGrant no 075-15-2019-1926. The second author was partially supported by RFBR, project number 20-31-70039 and the Council for the Support of Leading Scientific Schools of the President of the Russian Federation (grant no. N.Sh.-2540.2020.1). 


\section{References}

[B] B. Bollobás, On generalised graphs, Acta Math. Acad. Sci. Hungar 16 (1965), 447-452.

[EKR] P. Erdős, C. Ko, and R. Rado, Intersection theorems for systems of finite sets, The Quarterly Journal of Mathematics 12 (1961), 313--320.

[F1] P. Frankl, An extremal problem for two families of sets, European J. Combinatorics 3 (1982), 125-127.

[F2] P. Frankl, Erdős-Ko-Rado Theorem with conditions on the maximal degree, $J$. Combinatorial Theory A 46 (1987), 252-263.

[GLPPS] D. Gerbner, N. Lemons, C. Palmer, B. Patkós, and V. Szécsi, Almost intersecting families of sets, SIAM J. Discrete Math. 26 (2012), 1657-1699.

[H] A. J. W. Hilton, unpublished manuscript, 1976.

$[\mathrm{HM}] \quad$ A. J. W. Hilton and E. C. Milner, Some intersection theorems for systems of finite sets, Quart. J. Math. Oxford 18 (1967), 369-384.

[JP] F. Jaeger and C. Payan, Nombre maximal d'aretes d'un hypergraphe critique de rang h, CR Acad. Sci. Paris 273 (1971), 221-223

[Ka1] G. O. H. Katona, Solution of a problem of Ehrenfeucht and Mycielski, J. Combinatorial Theory A 17 (1974), 265-266.

[Ka2] G. O. H. Katona, A Theorem of Finite Sets, in: Theory of Graphs, Proc. Colloq. Tihany, 1966, Akadémiai Kiadó, 1968, pp. 187-207.

[Kr] J. B. Kruskal, The Number of Simplices in a Complex, in: Math. Optimization Techniques, California Press, Berkeley, 1963, 251-278.

[KZ] A. Kupavskii and D. Zakharov, Regular bipartite graphs and intersecting families, J. Combinatorial Theory A 155 (2018), 180-189. 